\title{
Primary Cutaneous Apocrine Carcinoma with Distant Metastasis
}

Titou Hicham*, Boui Mohammed and Hjira Naoufal

Dermatology and Venereology Department, Military Teaching Hospital Mohamed V, Mohammed V University, Rabat, Morocco

*Corresponding author: Titou Hicham, Resident, Dermatology and Venereology Department, Military Teaching Hospital Mohamed V, Mohammed $\mathrm{V}$ University, Rabat, Morocco, Tel: +212667191419; E-mail: titou.medic@gmail.com

Received date: November 13, 2018; Accepted date: November 27, 2018; Published date: December 15, 2018

Copyright: () 2018 Hicham T, et al. This is an open-access article distributed under the terms of the Creative Commons Attribution License, which permits unrestricted use, distribution, and reproduction in any medium, provided the original author and source are credited.

\begin{abstract}
Introduction: Cutaneous apocrine carcinomas (CAC) are rare adnexal malignant tumors preferentially localized in the axillary and anogenital regions. They are characterized by mammillated plaques or asymptomatic nodules and present a differential diagnosis problem with cutaneous metastases of breast adenocarcinoma. Metastatic CACs are a therapeutic challenge.
\end{abstract}

Case presentation: A 63-years-old man, chronic smoker, presented with painless left axillary mammillated plaques that had been slowly evolving for 18 months. Clinical examination found a tumor mass associated with magma of left axillary lymph nodes. Histological study and immunohistochemical staining of the skin evoked the diagnosis of cutaneous metastasis of breast adenocarcinoma. However, the diagnosis of axillary CAC was retained owing to the negativity of explorations in search of a primitive adenocarcinoma and the slow clinical course. Morphological assessment showed lymph node, hepatic and pulmonary metastases. Surgical excision with lymph node dissection was performed followed by chemotherapy.

Discussion: Metastatic CACs are rare. Sometimes, the existence of misleading tumor architecture and nonspecific nature of the immunolabeling explain the difficulty of the differential histological diagnosis between axillary $\mathrm{CAC}$ and cutaneous metastasis of lobular breast adenocarcinoma. Because of the rarity of this neoplasia no effective treatment is currently established.

Conclusion: We report a new case of metastatic axillary CAC. This case illustrates the importance of anatomoclinical correlation in cutaneous oncology and highlighted the need of treatment outcome evaluations of other cases of metastatic CAC to offer optimal treatment.

Keywords: Cutaneous apocrine carcinoma; Breast adenocarcinoma; Chemotherapy

\section{Introduction}

CACs are rare adnexal malignant tumors preferentially localized in the axillary and anogenital areas. Clinically, CACs are characterized by mammillated plaques or asymptomatic nodules. This neoplasia is slowly progressive, but may be locally aggressive or even metastatic, particularly at lungs, liver, brain and bones [1-3]. Prognosis is better in the absence of lymph node involvement and for non-metastatic forms [4]. CACs pose the problem of differential diagnosis with cutaneous metastasis of mammary adenocarcinoma. Because of its rarity, no chemotherapy protocol has been established for metastatic CACs. We report a new case of metastatic CAC, treated with chemotherapy, and we will highlight the diagnostic and therapeutic difficulties.

\section{Case Presentation}

A 63 year old chronic smoker patient was admitted for painless axillary mammillary plaques that have been progressively evolving for 18 months (Figure 1). Interrogation did not reveal any performance status change. Clinical examination found magma of left axillary lymphadenopathy. No right axillary mass or supernumerary nipples. Histopathological study of the left axillary cutaneous biopsy revealed intradermal tumor proliferation of small clusters and nests consisting of atypical cells of small to medium size, irregular, hyperchromatic round nucleus, and eosinophilic cytoplasm (Figure 2).

Exhaustive radiological assessment in search of a primitive adenocarcinoma was carried out. Thoraco-abdominopelvic CT showed left supraclavicular and left axillary centimetric adenomegaly, with multiple hepatic nodules (Figure 3).

Breast ultrasound was normal as well as bone scintigraphy and cerebral CT. Endoscopic digestive exploration and thyroid ultrasound were unremarkable.

Surgical excision of the axillary lesion with lymph node dissection was performed. Histological study results of the operative specimen were similar to the cutaneous biopsy: cutaneous metastasis of breast adenocarcinoma. All lymphadenopathies in the operative specimen were invaded by tumor proliferation with capsular intrusion. The internal margin of the resection was affected. No ectopic breast tissue has been detected. The immunohistochemical staining showed expression of cytokeratin 7 (CK7), GCDFP-15, estrogen and progesterone receptors by tumor cells (Figure 4). Tumor cells did not express the receptor for human epidermal growth factors (HER2) and p63 protein. 


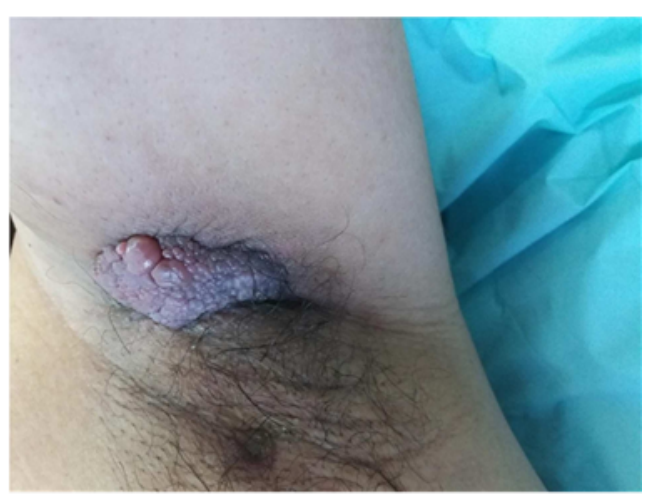

Figure 1: Carcinome apocrine cutanée axillaire. Axillary apocrine carcinoma of the skin.

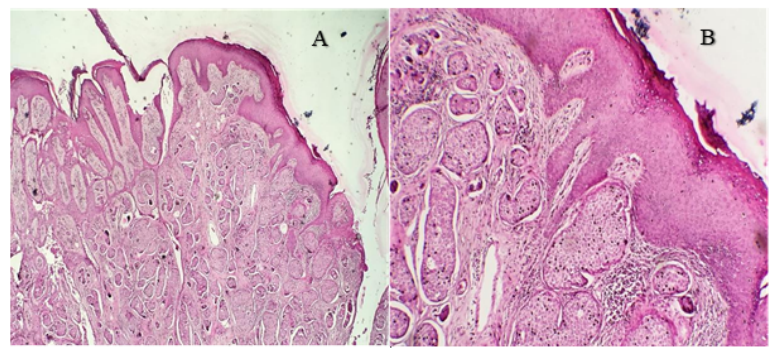

Figure 2: (A) Dermal coating in which the dermis is occupied by an invasive carcinomatous tumor process $(\mathrm{HE}, \mathrm{G} \times 50)$; (B) This process is made of clusters and nests of varying size $(\mathrm{HE}, \mathrm{G} \times 100)$.

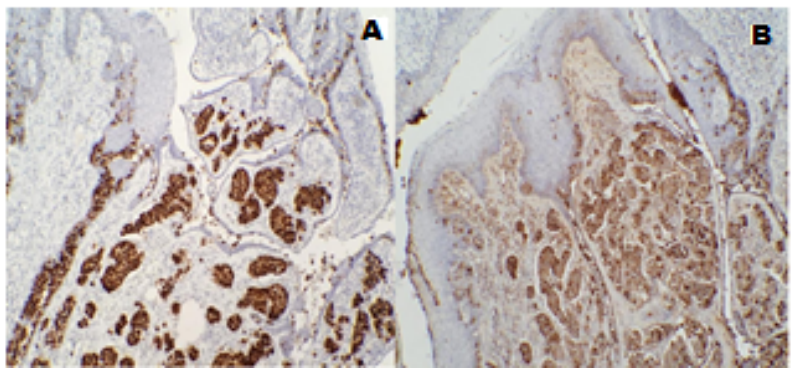

Figure 3: (A) Expression of progesterone receptors by tumor cells; (B) Expression of the GCDFP15 antibody by the tumor cells.

The diagnosis of metastatic axillary CAC (lymph node, hepatic and pulmonary metastases) was retained because of the following arguments: the clinical presentation, the slow clinical course of the tumor, and the negativity of complementary tests looking for primitive adenocarcinoma.

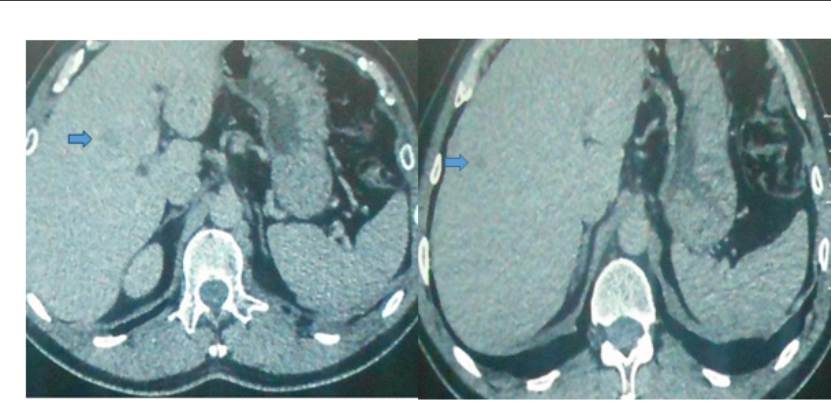

Figure 4: Hepatic nodules on CT scan.

The patient was treated with 6 chemotherapy courses of paclitaxel $175 \mathrm{mg} / \mathrm{m}^{2}$ combined with carboplatin AUC 5. The treatment was well tolerated with minimal adverse effects. At the end of treatment, the CT control showed a partial response of $39 \%$ according to the RECIST (Response Evaluation Criteria in Solid Tumors) criteria. Maintenance therapy with anti-estrogens (tamoxifen) was justified because of hormone receptors positivity. After 6 months, the CT scan control showed a stationary state. Currently, the patient is continuing treatment.

\section{Discussion}

Primary cutaneous apocrine carcinomas are rare malignant adnexal tumors that can be a diagnostic and therapeutic challenge. Skin areas rich in apocrine glands such as axillae and anogenital regions are the most common origin sites [5]. Other localizations are possible especially on the scalp, the eyelids, the upper lip, the face, and the nipple [6]. They are in the form of plaques or nodules with mammillated surfaces. Usually, they are asymptomatic and can reach large diameters and ulcerate.

CACs must always evoke the possibility of cutaneous metastases of lobular breast adenocarcinoma or digestive adenocarcinoma requiring a complete radiological and endoscopic assessment [7].

Histologically, these carcinomas are organized into tubular and papillary structures. The cells are typically eosinophilic and the diagnosis of apocrine tumors occurs in the presence of images of decapitation secretion within a cystic or tubular structure or papillary projections [8,9]. Immunohistochemical markers have not been shown to be completely reliable and sensitive in differentiating a breast tumor from a skin tumor. Indeed, there is a degree of variation in the expression of immunohistochemical markers that can aid diagnosis.

Although p63 is preferentially expressed in primary carcinomas of the sweat glands $[10,11], \mathrm{CACs}$ are typically negative for this marker [10]. GCDFP-15 is a marker of apocrine glandular differentiation but may still be positive in breast adenocarcinoma and in salivary gland carcinomas [12]. Positive immunostaining for GCDFP-15 is an insufficient argument. Anti-HER-2 immunolabeling is negative in apocrine primary skin carcinomas $[13,14]$. This marker is used because it is sometimes positive in metastases of breast adenocarcinoma $[13,14]$. Hormonal receptors are more frequently expressed in CAC than in lobular breast adenocarcinoma metastases [14]. In our case, the diagnosis of CAC was retained because of the clinical presentation, the slowly progressive clinical course and the negativity of paraclinical explorations in search of another primary adenocarcinoma. 
Most cases of localized CAC are managed by wide surgical excision, with margins of 1 to $2 \mathrm{~cm}$ (CK5-C3). The lymph node involvement and the presence of distant metastases have a poor impact on survival [4]. Patients with a metastatic CAC have a median survival of 14.5 months and a 5 years survival of $10 \%$ [4].

No effective treatment is currently established for metastatic CACs. Clinical trials remain difficult because of the rarity of this neoplasia.

The mammary glands are considered as modified apocrine glands. Almost half of mammary carcinomas present focal apocrine differentiation [15] with an immunohistochemical profile similar to CACs. This similarity justifies the use of NCCN recommendations for the management of breast carcinoma. According to these recommendations, anti-HER2 treatment can be used for tumors with HER2-over expressing. Hidaka et al. obtained a complete response for a case of metastatic HER2- positive CAC by treating the patient with anti-HER2 therapy [16]. For HER2-negative tumors, several chemotherapy protocols have been recommended. This is the case of our patient, who was treated with 6 courses of chemotherapy according to a protocol associating paclitaxel $\left(175 \mathrm{mg} / \mathrm{m}^{2}\right)$ with carboplatin AUC 5. At the end of the treatment, a partial response was objectified according to RECIST criteria. In our case, the positivity of hormonal receptors justified the use of hormonal treatment by Tamoxifen (anti-estrogen). A complete response with Tamoxifen has been reported in a patient with metastatic ER-positive apocrine carcinoma (CK18-C3). The follow-up will determine the medium and long-term evolution of our patient. Evaluation of treatment outcomes from other cases of metastatic CAC is needed to provide optimal treatment for this rare neoplasia.

\section{Conclusion}

Metastatic CACs are rare. They present a diagnostic and therapeutic challenge. This case report illustrates the importance of the anatomoclinical correlation in cutaneous oncology. It highlighted on the one hand, the interest of multidisciplinary care and long-term follow-up and, on the other hand, the need to evaluate treatment results of other metastatic CACs cases in order to provide optimal treatment.

\section{References}

1. Pai RR, Kini JR, Achar C, Rau A, Kini H (2008) Apocrine (cutaneous) sweat gland carcinoma of axilla with signet ring cells. A diagnostic dilemma on fine-needle aspiration cytology. Diagn Cytopathol 36: 739-741.
2. Tlemcani K, Levine D, Smith RV, Brandwein-Gensler M, Staffenberg DA, et al. (2010) Metastatic apocrine carcinoma of the scalp: prolonged response to systemic chemotherapy. J Clin Oncol 28: 412-414.

3. Gallerani E, Ciriolo M, Rossini C, Cavalli F (2007) Axillary apocrine carcinoma with brain metastases. J Clin Oncol 25: 5655-5656.

4. Hollowell KL, Agle SC, Zervos EE, Fitzgerald TL (2012) Cutaneous apocrine adenocarcinoma: defining epidemiology, outcomes, and optimal therapy for a rare neoplasm. J Surg Oncol 105: 415-419.

5. Robson A, Lazar AJ, Ben Naji J, Hanby A, Grayson W, et al. (2008) Primary cutaneous apocrine carcinoma: A clinico-pathologic analysis of 24 cases. Am J Pathol 32: 682-690.

6. Chamberlain R, Huber K, White J, Travaglino-Parda R (1999) Apocrine carcinoma of the axilla: Review of the literature and recommendations for treatment. Am J Clin Oncol 22: 131-135.

7. Misago N, Shinoda Y, Okawa T, Aoki S, Toda S, et al. (2011) Histiocytoid and signet-ring cell carcinoma of the axilla: a type of cutaneous apocrine carcinoma equivalent to histiocytoid lobular carcinoma of the breast? Clin Exp Dermatol 36: 874-877.

8. Warkel RL, Helwig EB (1978) Apocrine gland adenoma and adenocarcinoma of the axilla. Arch Dermatol 114: 198-203.

9. Paties C, Taccagni GL, Papotti M, Valente G, Zangrandi A (1993) Apocrine carcinoma of the skin. A clinicopathologic, immunocytochemical, and ultrastructural study. Cancer 71: 375-381.

10. Mahalingam M, Nguyen LP, Richards JE, Muzikansky A, Hoang MP (2010) The diagnostic utility of immunohistochemistry in distinguishing primary skin adnexal carcinomas from metastatic adenocarcinoma to skin: an immunohistochemical reappraisal using cytokeratin 15, nestin, p63, D2-40, and calretinin. Mod Pathol 23: 713-719.

11. Qureshi HS, Ormsby AH, Lee MW, Zarbo RJ, Ma CK (2004) The diagnostic utility of p63, CK5/6, CK7, and CK20 in distinguishing primary cutaneous adnexal neoplasms from metastatic carcinomas. J Cutan Pathol 31: 145-152.

12. Matsuo K, Fukutomi T, Hasegawa T, Akashi-Tanaka S, Nanasawa T, et al. (2002) Histological and immunohistochemical analysis of apocrine breast carcinoma. Breast Cancer 9: 43-49.

13. Hiatt KM, Pillow JL, Smoller BR (2004) Her-2 expression in cutaneous eccrine and apocrine neoplasms. Mod Pathol 17: 28-32.

14. Piris A, Peng Y, Boussahmain C, Essary LR, Gudewicz TM (2014) Cutaneous and mammary apocrine carcinomas have different immunoprofiles. Hum Pathol 45: 320-326.

15. Mazoujian G, Pinkus GS, Davis S, Haagensen DE Jr. (1983) Immunohistochemistry of a gross cystic disease fluid protein (GCDFP-15) of the breast. A marker of apocrine epithelium and breast carcinomas with apocrine features. Am J Pathol 110: 105-112.

16. Hidaka T, Fujimura T, Watabe A, Hashimoto A, Haga T, et al. (2012) Successful treatment of HER2-positive metastatic apocrine carcinoma of the skin with lapatinib and capecitabine. Acta Derm Venereol 92: 654-655. 